\title{
Impact of COVID-19 outbreak on patients with ST-segment elevation myocardial infarction (STEMI) in Turkey: results from TURSER study (TURKISH St-segment elevation myocardial infarction registry)
}

\author{
Tuncay Kiris ${ }^{1}$ Eyüp Avci ${ }^{2}$ Tuba Ekin ${ }^{3}$. Didar Elif Akgün ${ }^{2} \cdot$ Mücahit Tiryaki $^{1}$ - Arafat Yidirim ${ }^{4} \cdot$ Kutluhan Hazir $^{5}$. \\ Bektaş Murat ${ }^{6} \cdot$ Mehtap Yeni $^{7} \cdot$ Rojhad Altindag $^{8} \cdot$ Sefa Gül ${ }^{9} \cdot$ Baran Arik $^{10}$ - Tuncay Güzel $^{11}$. Selda Murat ${ }^{12}$. \\ Ahmet $\mathrm{Oz}^{13} \cdot$ Mustafa Karabacak $^{14} \cdot$ Zihni Aktas $^{15} \cdot$ Tarik Yildirim $^{2} \cdot$ Baris Kilicaslan $^{5} \cdot$ Asim Oktay Ergene $^{3}$
}

Accepted: 18 May 2021 / Published online: 29 May 2021

(c) The Author(s), under exclusive licence to Springer Science+Business Media, LLC, part of Springer Nature 2021

\begin{abstract}
Objective We aimed to investigate both the impact of COVID-19 pandemic on ST-segment elevation myocardial infarction (STEMI) admission, and demographic, angiographic, procedural characteristics, and in-hospital clinical outcomes of patients with COVID-19 positive STEMI in Turkey.

Methods This was a multi-center and cross-sectional observational study. The study population included 1788 STEMI patients from 15 centers in Turkey. The patients were divided into two groups: COVID-19 era (March 11st-May 15st, 2020; $\mathrm{n}=733$ ) or pre- COVID-19 era group (March 11st-May 15st, 2019; $\mathrm{n}=1055$ ). Also, the patients in COVID-19 era were grouped as COVID-19 positive $(n=65)$ or negative $(n=668)$.

Results There was a 30.5\% drop in STEMI admission during COVID-19 era in comparison to pre-COVID-19 era. The patients admitted to the medical centers during COVID-19 era had a longer symptom-to-first medical contact time [120 (75-240) vs. 100 (60-180) minutes, $\mathrm{p}<0.001]$. COVID-19 positive STEMI patients had higher thrombus grade and lower left ventricular ejection fraction compared to COVID-19 negative patients. COVID-19 positive patients had higher mortality $(28 \%$ vs. $6 \%, \mathrm{p}<0.001)$ and cardiogenic shock $(20 \%$ vs. $7 \%, \mathrm{p}<0.001)$ rates compared with those without COVID-19. Matching based on propensity scores showed higher mortality and high thrombus grade in STEMI patients who were infected by SARS-COV-2 (each $\mathrm{p}<0.05$ ).

Conclusions We detected significantly lower STEMI hospitalization rates and significant delay in duration of symptom onset to first medical contact in the context of Turkey during the COVID-19 outbreak. Moreover, high thrombus grade and mortality were more common in COVID-19 positive STEMI patients.
\end{abstract}

Keywords COVID-19 - SARS-CoV2 $\cdot$ ST elevation myocardial infarction · Primary percutaneous coronary $\cdot$ Intervention $\cdot$ Mortality

\section{Highlights}

- There was a significantly reduced STEMI admission in COVID-19 era compared with pre-COVID-19 era.

- COVID+ patients with ST-Elevation represent a unique and high-risk patient population.

Tuncay Kiris

drtkiris@hotmail.com

Extended author information available on the last page of the article
- COVID+ patients with ST-Elevation had higher in-hospital mortality and thrombus grade.

- No differences in culprit vessel and similar symptomonset to first medical contact times between COVID-19 $(+)$ and COVID-19 (-).

- COVID+ patients with ST-Elevation were more likely to not receive angiography (5\%) and to receive medical therapy but still $95 \%$ received PPCI and lytics were uncommon, thus primary PCI is preferable (and feasible) in COVID+ patients. 


\section{Introduction}

The COVID-19 outbreak that has developed due to the new type of corona virus has caused thousands of deaths in the world [1]. COVID-19, the highly infectious disease caused by a novel coronavirus, killed more than a million people globally. Our country has been affected from this epidemic to a certain extent. There may be changes in the hospital admission pattern for other conditions such as acute coronary syndrome, heart failure during this period [2]. It has been shown that reducing delay in reperfusion treatment in ST-segment elevation myocardial infarction (STEMI) patients is associated with improved outcomes in many studies $[3,4]$. Little has been known about the impact of outbreak of infectious disease on pre- and in-hospitallogistics of STEMI care, especially in patient-related delays.

Coagulation abnormalities and tendency to thrombotic disease have been shown to be more common in patients with coronavirus disease 2019 (COVID-19) due to increased inflammation, platelet activation, endothelial dysfunction [5]. The data on the impact of COVID-19 on thrombus burden, in-hospital outcomes including mortality, cardiojenic shock in STEMI patients is inadequate [6].

We aimed to evaluate both impact of the COVID-19 pandemic on patients with STEMI admission and demographic, angiographic, and procedural characteristics as well as inhospital clinical outcomes of patients with COVID-19 positive STEMI in Turkey.

\section{Methods}

\section{Study design and patient population}

This is a multicenter, retrospective, observational study that included 1788 patients, between 18 and 90 years of age, diagnosed with STEMI in 15 centers (Fig. 1). The patients were divided into two groups: COVID-19 era (March 11st-May 15st, 2020; $\mathrm{n}=733$ ) and pre- COVID-19 era group (March 11st-May 15st, 2019; $\mathrm{n}=1055$ ). Moreover, the patients in COVID-19 era were grouped as COVID-19 positive $n=65)$ or negative $(n=668)$. STEMI was defined according to the fourth universal definition of myocardial infarction [7]. Evidence-based optimal medical therapy and coronary revascularization according to current guidelines and recommendations were performed [8]. All interventional procedures and strategies such as balloon angioplasty, type of stent, aspiration thrombectomy, and the usage of intraaortic balloon pump were left to the discretion of interventional cardiologists. The thrombus burden can be grouped based on the thrombolysis in myocardial infarction (TIMI) thrombus grade [9]. Patients with Grade 5 thrombus burden were reclassified to a thrombus category after flow achievement either with a guidewire or a small $(1.5 \mathrm{~mm})$ balloon [10]. The left ventricle ejection fraction (LVEF) was calculated after measuring the end-diastolic and end-systolic left ventricle (LV) volumes in the apical four-chamber and two-chamber views using the modified Simpson's method. Valve disease was defined as moderate or severe regurgitation in mitral or aortic valves, or severe stenosis for mitral or aortic valves.

Bleeding classification was performed according to the TIMI bleeding score. Major hemorrhage was defined as $5 \mathrm{gr} / \mathrm{dl}$ hemogram, a $15 \%$ or greater decline in hematocrit, or intracranial hemorrhage. Minor bleeding was defined as $3-5 \mathrm{gr} / \mathrm{dl} \mathrm{Hb}$, a $10-15 \% \mathrm{gr} / \mathrm{dl}$ hematocrit decline, or gastrointestinal bleeding. Cardiogenic shock was defined as systolic blood pressure $<90 \mathrm{mmHg}$ for at least $30 \mathrm{~min}$ with evidence of poor tissue perfusion after correction of non-myocardial factors.

After the STEMI diagnosis, the patients with no signs of COVID-19 infection were transferred to the routine cardiac catheterization laboratory, however the patients with symptoms indicating possible COVID-19 were transferred to an allocated cardiac catheterization laboratory.

The diagnosis of COVID-19 was confirmed by detecting SARS-CoV-2 on nasal/pharyngeal swab [11] or by evaluating the semptoms plus radiological imaging [12]. All of these patients were treated as COVID-19 patients in these centers. The study was approved by the ethics committee of the Dokuz Eylul University Faculty of Medicine (2020/1035) and Ministry of Health (2020-05-02T23_17_42).

\section{Data collection}

The patient's data including hypertension (HT), diabetes mellitus (DM), chronic obstructive pulmonary disease (COPD) or asthma, previous coronary artery disease (CAD), COVID-19 status, shock, in-hospital mortality, stent thrombosis, blood samples, thrombus aspiration device use, glycoprotein IIb/IIIa inhibitors use, echocardiographic, and angiographic imagings from each center were collected by principial investigator of that center. Cineangiographic images of patients were retrospectively analysed by two interventional cardiologist blinded to the patient's COVID19 status. Data were finally checked for missing or contradictory entries.

\section{Study outcomes}

The main outcome was the reduction in admission rates in STMI patients. Moreover, in-hospital outcomes included cardiogenic shock and mortality were compared between two groups. Also, thrombus grade, mortality, and cardiogenic 
shock rates were evaluated in COVID-19 positive STEMI patients.

\section{Statistical analysis}

Categorical variables are provided as absolute numbers, percentages, and compared by the $\mathrm{x}^{2}$ test. Continuous variables are presented as mean and Standard deviation (SD) and compared by the Student's t-test, or Mann-Whitney test as appropriate. We used a propensity score matching method to balance the confounding effect due to differences in characteristics between the COVID1-9 positive group and the nonCOVID-19 group because of the retrospective nature of the study. We estimated a propensity score to create a matched dataset (COVID-19 vs. non-COVID-19) and the covariates including age, symptoms at admission, gender, COPD or asthma, LVEF, valve disease, LV wall motion anormality, coronary intervention or patients treated with medical treatment, use of Glycoprotein IIb/IIIa inhibitors, thrombus aspiration device, complete revascularization during the index hospitalization, WBC, troponin, and C-reactive protein levels were considered for balance between COVID-19 positive and non-COVID-19. Two-sided p-values $<0.05$ were considered statistically significant. All statistical analysis was performed with SPSS version 25 (SPSS Inc., Chicago, IL, USA) and R software.
Fig. 1 Flow diagram of patient recruitment
Initially, 16 centers were included in the study $(n=1890)$

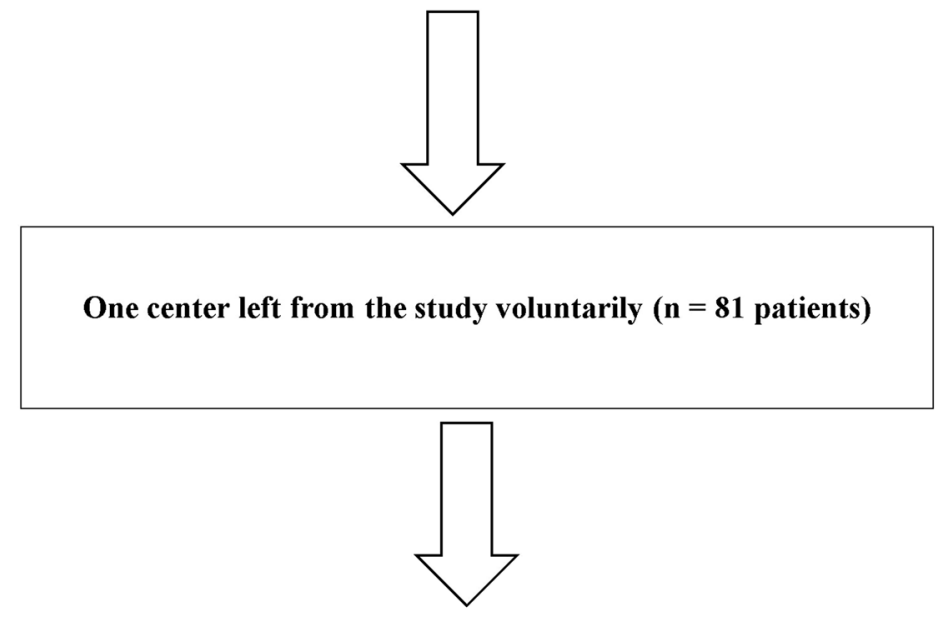

21 patients, who had incomplete data or were transferred to other medical facilities prevented the collection of outcome data, were excluded from the study

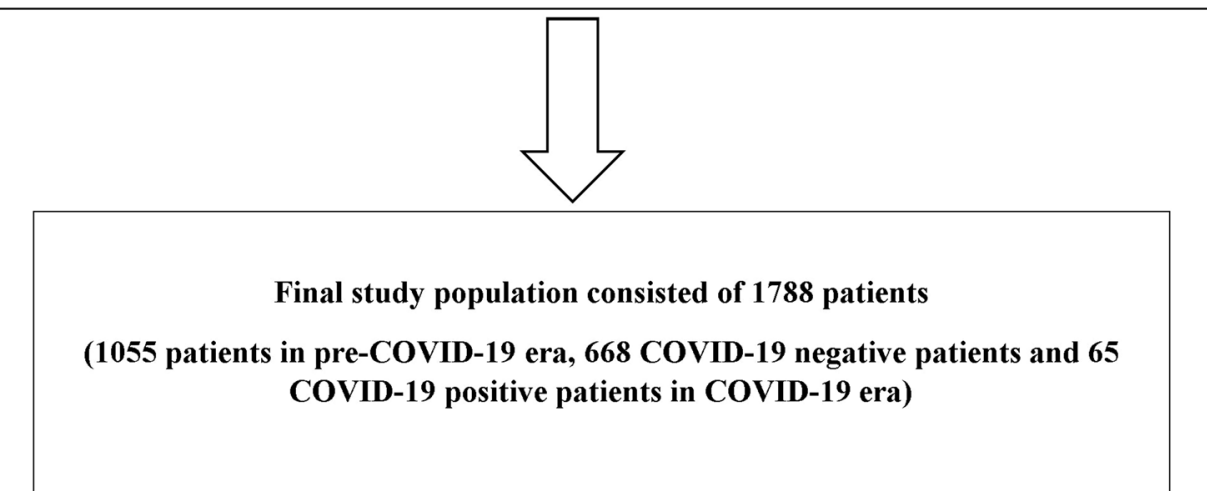


Table 1 Baseline characteristics of the study population

\begin{tabular}{|c|c|c|c|}
\hline Variables & $\begin{array}{l}\text { Pre-COVID-19 } \\
\text { era }(n=1055)\end{array}$ & COVID-19 era $(n=733)$ & p-value \\
\hline STEMI admission (per/day) & 16.5 & 11.5 & $<0.001$ \\
\hline Age, years & $61.9 \pm 12.4$ & $60.6 \pm 12.4$ & 0.029 \\
\hline Symptoms at admission $\mathrm{n}(\%)$ & & & 0.004 \\
\hline Chest pain & $791(75)$ & $495(68)$ & \\
\hline Dyspnea & 198 (19) & $168(23)$ & \\
\hline Arrest & $10(1)$ & $9(1)$ & \\
\hline Other & $56(5)$ & $61(8)$ & \\
\hline Female gender $(\%)$ & $261(25)$ & $167(23)$ & 0.340 \\
\hline Hypertension, n (\%) & $400(38)$ & $309(42)$ & 0.071 \\
\hline Diabetes mellitus, n (\%) & $311(30)$ & $211(29)$ & 0.456 \\
\hline Previous AF, n (\%) & $46(4)$ & $39(5)$ & 0.348 \\
\hline Smoking, n (\%) & $325(31)$ & $244(33)$ & 0.268 \\
\hline Astım or COPD, n (\%) & $110(10)$ & $71(10)$ & 0.610 \\
\hline Previous CAD, n (\%) & $131(12)$ & $93(13)$ & 0.865 \\
\hline COVID-19 positive $\mathrm{n}(\%)$ & - & $65(9)$ & - \\
\hline \multicolumn{4}{|l|}{ Echocardiographic findings } \\
\hline $\operatorname{LVEF}(\%)$ & $47.9 \pm 9.1$ & $46.7 \pm 8.8$ & 0.005 \\
\hline LVWM abnormalities n (\%) & $653(62)$ & $492(67)$ & 0.024 \\
\hline Valve disease n (\%) & $106(10)$ & $66(9)$ & 0.618 \\
\hline Coronary intervention n (\%) & $1054(100)$ & $729(99)$ & 0.076 \\
\hline Infarct related artery $\mathrm{n}(\%)$ & & & 0.215 \\
\hline LMCA & $15(1)$ & $17(2)$ & \\
\hline LAD & $403(38)$ & $307(42)$ & \\
\hline $\mathrm{CX}$ & 199 (19) & $134(18)$ & \\
\hline RCA & $343(33)$ & $222(31)$ & \\
\hline Other & $85(8)$ & $43(6)$ & \\
\hline Noncritical CAD & $9(1)$ & $6(1)$ & \\
\hline Multi-vessel disease n (\%) & $421(40)$ & $294(40)$ & 0.870 \\
\hline Glycoprotein IIb/IIIa inhibitors n (\%) & $239(23)$ & $161(22)$ & 0.715 \\
\hline Thrombus aspiration device $\mathrm{n}(\%)$ & $61(6)$ & $56(8)$ & 0.121 \\
\hline IABP n $(\%)$ & $29(3)$ & $12(2)$ & 0.122 \\
\hline \multicolumn{4}{|l|}{ Baseline TIMI flow n (\%) } \\
\hline TIMI flow $0-1$ & $959(91)$ & $652(90)$ & 0.276 \\
\hline Baseline thrombus grade $>3$ & $711(68)$ & $499(69)$ & 0.659 \\
\hline Modified thrombus grade $>3$ & $434(42)$ & $296(41)$ & 0.684 \\
\hline Post-PCI TIMI 3 flow n (\%) & $878(83)$ & $594(82)$ & 0.319 \\
\hline Multi-vessel PCI during the index prodecure $\mathrm{n}(\%)$ & $98(9)$ & $71(10)$ & 0.778 \\
\hline $\begin{array}{l}\text { Complete revascularization during the index hospi- } \\
\text { talization } \mathrm{n}(\%)\end{array}$ & $190(18)$ & 127 (17) & 0.710 \\
\hline \multicolumn{4}{|l|}{ Previous medication } \\
\hline ACE-I/ARB, n (\%) & $265(25)$ & $205(28)$ & 0.138 \\
\hline Statin n (\%) & $181(17)$ & $114(16)$ & 0.369 \\
\hline Aspirin n (\%) & $246(24)$ & $208(28)$ & 0.008 \\
\hline $\mathrm{P} 2 \mathrm{Y} 12 \mathrm{Y}$ inhibitors & & & 0.019 \\
\hline Clopidogrel n (\%) & $187(76)$ & $133(64)$ & \\
\hline Tigacrelor n (\%) & $53(22)$ & $67(32)$ & \\
\hline Prasuqrel n (\%) & $6(2)$ & $8(4)$ & \\
\hline In hospital or discharge ASA + $\mathrm{P} 2 \mathrm{Y} 12 \mathrm{Y}$ inhibitors & & & 0.096 \\
\hline ASA plus Clopidogrel n (\%) & $779(74)$ & $502(69)$ & \\
\hline ASA plus Tigacrelor n (\%) & $180(17)$ & $148(20)$ & \\
\hline
\end{tabular}


Table 1 (continued)

\begin{tabular}{|c|c|c|c|}
\hline Variables & $\begin{array}{l}\text { Pre-COVID-19 } \\
\text { era }(n=1055)\end{array}$ & COVID-19 era $(n=733)$ & p-value \\
\hline ASA plus Prasuqrel n (\%) & $95(9)$ & $75(10)$ & \\
\hline Pharmaco-invazive treatment $\mathrm{n}(\%)$ & $9(1)$ & $4(1)$ & 0.452 \\
\hline Patients treated with medical treatment $\mathrm{n}(\%)$ & $4(0)$ & $7(1)$ & 0.126 \\
\hline Symptom-to-FMC, minutes (median [IQR]) & $100(60-180)$ & $120(75-240)$ & $<0.001$ \\
\hline Symptom-to- (FMC) time & & & $<0.001$ \\
\hline Less than $2 \mathrm{~h}, \mathrm{n}(\%)$ & $565(54)$ & $316(43)$ & \\
\hline 2 to $6 \mathrm{~h}, \mathrm{n}(\%)$ & $394(37)$ & $285(39)$ & \\
\hline 6 to $12 \mathrm{~h}, \mathrm{n}(\%)$ & $53(5)$ & $67(9)$ & \\
\hline 12 to $24 \mathrm{~h}, \mathrm{n}(\%)$ & $19(2)$ & $26(4)$ & \\
\hline More than $24 \mathrm{~h}, \mathrm{n}(\%)$ & $24(2)$ & $39(6)$ & \\
\hline \multicolumn{4}{|l|}{ In-hospital outcomes } \\
\hline Minor bleeding $\mathrm{n}(\%)$ & $29(3)$ & $16(2)$ & 0.455 \\
\hline Major bleeding n (\%) & $0(0)$ & $3(0.4)$ & 0.037 \\
\hline Stent thrombosis $\mathrm{n}(\%)$ & $15(1)$ & $12(2)$ & 0.713 \\
\hline Cardiogenic shock, $\mathrm{n}(\%)$ & $92(9)$ & $62(9)$ & 0.846 \\
\hline Mortality, n (\%) & $71(7)$ & $61(8)$ & 0.205 \\
\hline
\end{tabular}

$A C E-I / A R B$ angiotensin converting enzyme inhibitors/ angiotensin receptor blocker, $A F$ atrial fibrillation, $C A D$ : coronary artery disease, $C O P D$ chronic obstructive pulmonary disease, $F M C$ first medical contact, $I A B P$ intra aortic baloon pump, $I Q R$ inter quartile range, $L V E F$ left ventricular ejection fraction, $L V W M$ left ventricular wall motion abnormalities, PCI percutaneous coronary intervention, STEMI ST-segment elevation myocardial infarction

\section{Sample size calculation}

We did not perform sample size calculations or statistical power analyses due to the observational nature of this study. However, with the participation of at least 15 centers, with approximately 45 patients per center according to the average PPCI per month, we expected the inclusion of at least 700 patients with STEMI for each period.

\section{Results}

\section{Patient characteristics}

A total of 1788 STEMI admissions were noticed. The baseline characteristics of the study patients are provided in Table 1. As shown in Fig. 2, a 30.5\% decline in STEMI volume during COVID-19 era compared with the equivalent duration in pre-COVID-19 era. Daily STEMI admissions were reduced from 16.5 in 2019 to 11.5 in 2020 . Average age of patients in pre-COVID-19 era group was higher than
Fig. 2 Comparison of ST elevation hospitalizations in COVID19 era and pre-COVID-19 era

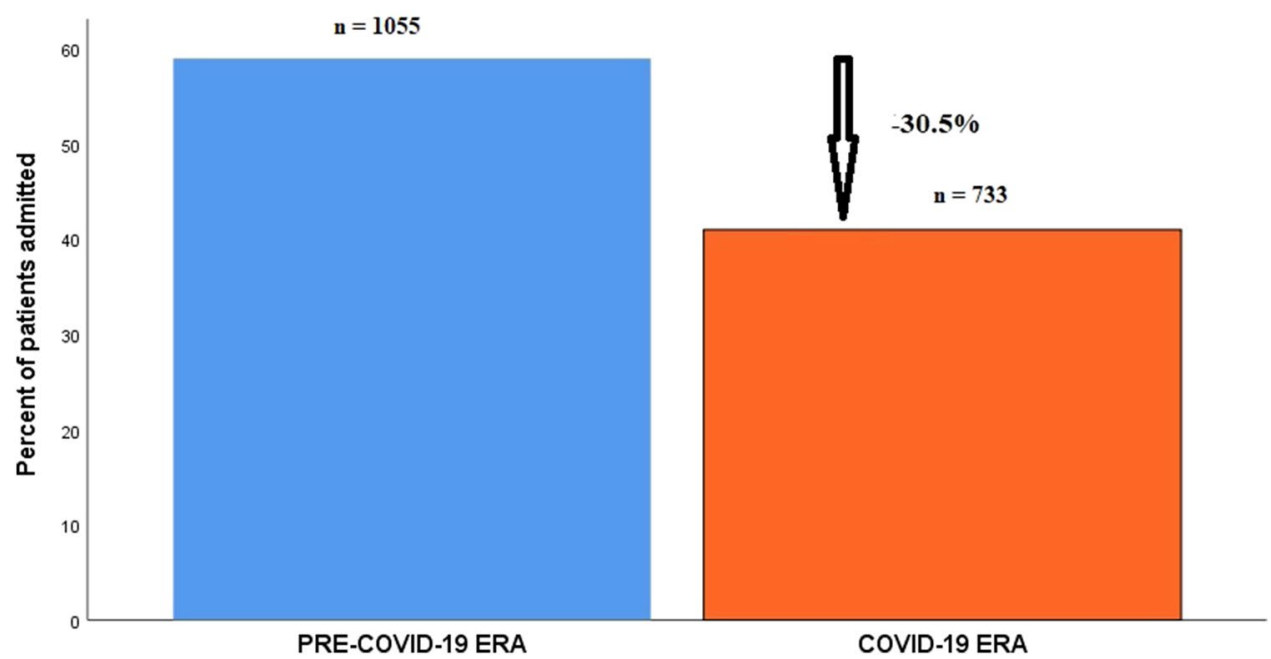




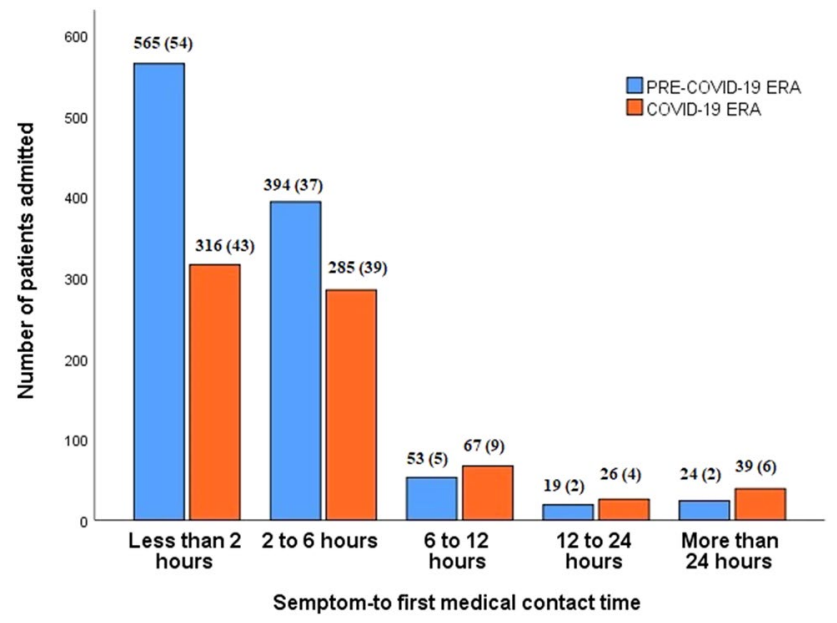

Fig. 3 Symptoms- to first medical contact time (FMC) for acute STsegment elevation. myocardial infarction (STEMI)

those in COVID-19 era group $(61.9 \pm 12.4$ vs $60.6 \pm 12.4$, $\mathrm{p}=0.029$ ).

There were no significant differences between groups regarding the history of diabetes mellitus, hypertension, coronary artery disease, and atrial fibrillation (Table 1). Also, the rates of pre-usage statin, and ACE-I angiotensinconverting enzyme inhibitors or angiotensin receptor blocker (ARB) were similar in both groups, whereas previous uses of aspirin or P2Y12Y inhibitors were less (Table 2). LVEF was lower in COVID-19 era patients compared to the preCOVID-19 era $(46.7 \pm 8.8$ vs. $47.9 \pm 9.1, \mathrm{p}=0.005)$. The number of patients who received pharmaco-invasive treatment was similar in both groups ( $1 \%$ vs. $1 \%, \mathrm{p}=0.452)$. The rate of coronary intervention did not differ between groups (100\% vs. $99 \%, \mathrm{p}=0.076)$.

Symptom-to- FMC time was longer in COVID-19 group than in pre-COVID-19 group [120 (75-240) vs. 100 (60-180) minutes, $\mathrm{p}<0.001]$. The duration was less than $2 \mathrm{~h}$ in $43 \%, 2$ to $6 \mathrm{~h}$ in $39 \%, 6$ to $12 \mathrm{~h}$ in $9 \%, 12$ to $24 \mathrm{~h}$ in $4 \%$, and more than $24 \mathrm{~h}$ in $6 \%$ of patients in COVID-19 era. The corresponding percentages in pre-COVID group were 54\%, $37 \%, 5 \%, 2 \%$, and $2 \%$, respectively (Fig. 3). The laboratory variables of the groups are shown in Table 2. There was no significant difference between groups with regard to hemoglobin, platelet counts, white blood cell counts (WBC), and serum creatinine levels (each $\mathrm{p}>0.05$ ). However, troponin levels were higher in COVID-19 era compared with preCOVID era [10100 (888-25000) vs. 5608.9 (346-19533), $\mathrm{p}=0.027]$.

In COVID-19 era, 65 STEMI patients were COVID-19 positive. The features of these patients were provided in Table 4. Compared with non-COVID-19, COVID-19 group was older $(66.8 \pm 12.0$ vs. $60.0 \pm 12.3, \mathrm{p}<0.001)$. There was no significant difference between groups regarding to the symptom onset to FMC time, histories of DM, HT, and CAD (Table 4). COPD or asthma was more common in COVID-19 positive patients when compared to those without COVID-19 (17\% vs. 9\%, $p=0.039$ ). Valve disease, left ventricular wall motion abnormalities, and low LVEF were more frequent in COVID-19 positive STEMI patients than those without (Table 3).

Patients with COVID-19 had higher levels of CRP, WBC, and troponin [55.4 (21.3-147.8) vs. $21.1(2.8-51.4)$, $\mathrm{p}<0.001 ; 13.6 \pm 4.8$ vs. $12.0 \pm 4.5, \mathrm{p}=0.007 ; 19,578$ (6762-27021) vs. 8388 (795-23600), p $=0.002$, respectively, Table 4] than those without COVID-19.

\section{Procedural characteristics}

There were no differences in infarct related artery, multivessel disease, glycoprotein IIb/IIIa inhibitors use, IABP, and thrombus aspiration device use between COVID-19 era and pre-COVID-19 era (Table 1). Also, baseline TIMI 0/1 flow, modified thrombus grade $>3$, and post-PCI TIMI -3 flow were similar in both groups Table 2, Fig 4.

The rate of coronary intervention was lower in patients with COVID-19 than those without (95\% vs. $100 \%$, $\mathrm{p}<0.001)$. Baseline modified thrombus grade $>3$ was higher COVID-19 group than in non-COVID-19 group (58\% vs. $39 \%, p=0.003$, Fig. 5). In parallel with this, thrombus aspiration device, and glycoprotein IIb/IIIa inhibitors uses were greater in patients with COVID-19 (14\% vs. $7 \%, \mathrm{p}=0.048 ; 34 \%$ vs. $21 \%, \mathrm{p}=0.015$, respectively)
Table 2 Laboratory findings of patients

\begin{tabular}{lllc}
\hline Variable & Pre-COVID-19 era $(\mathrm{n}=1055)$ & COVID-19 era $(\mathrm{n}=733)$ & $\mathrm{p}$-value \\
\hline WBC $\left(\times 10^{3} / \mu \mathrm{L}\right)$ & $11.8 \pm 3.9$ & $12.1 \pm 4.6$ & 0.143 \\
Hemoglobin $(\mathrm{mg} / \mathrm{dl})$ & $13.8 \pm 2.1$ & $13.9 \pm 2.1$ & 0.162 \\
Creatinine* $(\mathrm{mg} / \mathrm{dl})$ & $0.90(0.76-1.10)$ & $0.90(0.74-1.04)$ & 0.425 \\
Platelet $\left(\times 10^{9} / \mathrm{L}\right)$ & $256.3 \pm 75.2$ & $252.2 \pm 73.6$ & 0.253 \\
C-reactive protein* $(\mathrm{mg} / \mathrm{L})$ & $20(2.2-48.1)$ & $24.4(3.2-54.3)$ & 0.280 \\
Troponin* $(\mathrm{ng} / \mathrm{L})$ & $5608.9(346-19,533)$ & $10,100(888-25,000)$ & 0.027 \\
\hline
\end{tabular}

$W B C$ white blood cell

*Comparison was made using Mann-Whitney $U$ test at $P<0.05$, and these values were described by median with inter-quartile range (25th and 75 th percentile) 
Table 3 Baseline characteristics of the study population

\begin{tabular}{|c|c|c|c|}
\hline Variables & Non-COVID-19 $(n=668)$ & COVID-19 $(n=65)$ & p-value \\
\hline Age, years & $60.0 \pm 12.3$ & $66.8 \pm 12.0$ & $<0.001$ \\
\hline Symptoms at admission n (\%) & & & 0.004 \\
\hline Chest pain & $459(69)$ & $36(55)$ & \\
\hline Dyspnea & $153(23)$ & $15(23)$ & \\
\hline Arrest & $8(1)$ & $1(2)$ & \\
\hline Other & $48(7)$ & $13(20)$ & \\
\hline Female gender $(\%)$ & $142(22)$ & $21(32)$ & 0.055 \\
\hline Hypertension, n (\%) & $278(42)$ & $31(48)$ & 0.344 \\
\hline Diabetes mellitus, n (\%) & $193(29)$ & $18(26)$ & 0.851 \\
\hline Previous AF, n (\%) & $36(5)$ & $3(5)$ & 0.791 \\
\hline Smoking, n (\%) & $222(33)$ & $22(34)$ & 0.920 \\
\hline COPD or asthma, n (\%) & $60(9)$ & $11(17)$ & 0.039 \\
\hline Previous CAD, n (\%) & $81(12)$ & $12(19)$ & 0.143 \\
\hline \multicolumn{4}{|l|}{ Echocardiographic findings } \\
\hline $\operatorname{LVEF}(\%)$ & $47.0 \pm 8.8$ & $43.7 \pm 8.8$ & 0.004 \\
\hline LVWM abnormalities n (\%) & $441(68)$ & $51(82)$ & 0.041 \\
\hline Valve disease n $(\%)$ & $53(8)$ & $13(20)$ & 0.010 \\
\hline Coronary intervention $\mathrm{n}(\%)$ & $667(100)$ & $62(95)$ & $<0.001$ \\
\hline Infarct related artery $\mathrm{n}(\%)$ & & & 0.418 \\
\hline LMCA & $16(2)$ & $1(2)$ & \\
\hline LAD & $282(42)$ & $25(40)$ & \\
\hline $\mathrm{CX}$ & $119(18)$ & $15(24)$ & \\
\hline RCA & $208(31)$ & $4(23)$ & \\
\hline Other & $37(6)$ & $6(10)$ & \\
\hline Noncritical CAD & $5(1)$ & $1(2)$ & \\
\hline Multi-vessel disease n (\%) & $267(40)$ & $27(44)$ & 0.589 \\
\hline Glycoprotein IIb/IIIa inhibitors n (\%) & $139(21)$ & $22(34)$ & 0.015 \\
\hline Thrombus aspiration device $\mathrm{n}(\%)$ & $47(7)$ & $9(14)$ & 0.048 \\
\hline IABP n $(\%)$ & $10(2)$ & $2(3)$ & 0.338 \\
\hline \multicolumn{4}{|l|}{ Baseline TIMI flow n (\%) } \\
\hline TIMI flow $0-1$ & $594(89)$ & $58(94)$ & 0.271 \\
\hline Baseline thrombus grade $>3$ & $440(66)$ & $59(95)$ & $<0.001$ \\
\hline Modified thrombus grade $>3$ & $260(39)$ & $36(58)$ & 0.003 \\
\hline Post-PCI TIMI 3 flow n (\%) & $544(82)$ & $50(81)$ & 0.859 \\
\hline Multi-vessel PCIduring the index prodecure $\mathrm{n}(\%)$ & $66(10)$ & $5(8)$ & 0.569 \\
\hline $\begin{array}{l}\text { Complete revascularization during the index hospitalization } \\
\mathrm{n}(\%)\end{array}$ & $109(16)$ & $18(29)$ & 0.021 \\
\hline \multicolumn{4}{|l|}{ Previous medication } \\
\hline ACE-I/ARB, n (\%) & $186(28)$ & $19(29)$ & 0.812 \\
\hline Statin n $(\%)$ & $100(15)$ & $14(22)$ & 0.163 \\
\hline Aspirin n (\%) & $185(28)$ & $23(35)$ & 0.189 \\
\hline $\mathrm{P} 2 \mathrm{Y} 12 \mathrm{Y}$ inhibitors & & & 0.220 \\
\hline Clopidogrel n (\%) & $122(66)$ & $11(48)$ & \\
\hline Tigacrelor n (\%) & $56(30)$ & $11(48)$ & \\
\hline Prasuqrel n (\%) & $7(4)$ & $1(4)$ & \\
\hline In hospital or discharge ASA $+\mathrm{P} 2 \mathrm{Y} 12 \mathrm{Y}$ inhibitors & & & 0.664 \\
\hline ASA plus Clopidogrel n (\%) & $465(70)$ & $37(64)$ & \\
\hline ASA plus Tigacrelor $\mathrm{n}(\%)$ & $134(20)$ & $14(24)$ & \\
\hline ASA plus Prasuqrel n (\%) & $68(10)$ & $7(12)$ & \\
\hline Pharmaco-invazive treatment $\mathrm{n}(\%)$ & $3(1)$ & $1(2)$ & 0.255 \\
\hline
\end{tabular}


Table 3 (continued)

\begin{tabular}{|c|c|c|c|}
\hline Variables & Non-COVID-19 $(n=668)$ & COVID-19 $(n=65)$ & p-value \\
\hline Patients treated with medical treatment n (\%) & $3(0)$ & $4(6)$ & $<0.001$ \\
\hline Symptom-to-FMC, minutes (median [IQR]) & $120(75-240)$ & $120(78-240)$ & 0.842 \\
\hline Symptom-to- (FMC) time & & & 0.606 \\
\hline Less than $2 \mathrm{~h}, \mathrm{n}(\%)$ & $289(43)$ & $27(42)$ & \\
\hline 2 to $6 \mathrm{~h}, \mathrm{n}(\%)$ & $255(38)$ & $30(46)$ & \\
\hline 6 to $12 \mathrm{~h}, \mathrm{n}(\%)$ & $64(10)$ & $3(5)$ & \\
\hline 12 to $24 \mathrm{~h}, \mathrm{n}(\%)$ & $24(4)$ & $2(3)$ & \\
\hline More than 24 h, n (\%) & $36(5)$ & $3(5)$ & \\
\hline \multicolumn{4}{|l|}{ In-hospital outcomes } \\
\hline Minor bleeding n (\%) & $14(2)$ & $2(3)$ & 0.591 \\
\hline Major bleeding n (\%) & $3(0)$ & $0(0)$ & 0.591 \\
\hline Stent thrombosis n (\%) & $8(1)$ & $4(6)$ & 0.002 \\
\hline Cardiogenic shock, n (\%) & $49(7)$ & $13(20)$ & $<0.001$ \\
\hline Mortality, n (\%) & $43(6)$ & $18(28)$ & $<0.001$ \\
\hline
\end{tabular}

$A C E-I / A R B$ angiotensin converting enzyme inhibitors/angiotensin receptor blocker, $A F$ atrial fibrillation, $C A D$ coronary artery disease, $C O P D$ chronic obstructive pulmonary disease, $F M C$ first medical contact, $I A B P$ intra aortic balon pump, IQR inter quartile range, $L V E F$ left ventricular ejection fraction, $L V W M$ left ventricular wall motion abnormalities, $P C I$ percutaneous coronary intervention, STEMI ST-segment elevation myocardial infarction

Table 4 Laboratory findings of patients

\begin{tabular}{lllr}
\hline Variable & Non-COVID-19 $(\mathrm{n}=668)$ & COVID-19 $(\mathrm{n}=65)$ & p-value \\
\hline WBC $\left(\times 10^{3} / \mu \mathrm{L}\right)$ & $12.0 \pm 4.5$ & $13.6 \pm 4.8$ & 0.007 \\
Hemoglobin $(\mathrm{mg} / \mathrm{dl})$ & $13.9 \pm 2.2$ & $13.6 \pm 2.3$ & 0.229 \\
Creatinine* $(\mathrm{mg} / \mathrm{dl})$ & $0.90(0.73-1.03)$ & $0.90(0.80-1.21)$ & 0.936 \\
Platelet $\left(\times 10^{9} / \mathrm{L}\right)$ & $251.8 \pm 72.2$ & $256.3 \pm 87.1$ & 0.645 \\
C-reactive protein* $(\mathrm{mg} / \mathrm{L})$ & $21.1(2.8-51.4)$ & $55.4(21.3-147.8)$ & $<0.001$ \\
Troponin* $(\mathrm{ng} / \mathrm{L})$ & $8388(795-23,600)$ & $19,578(6762-27,021)$ & 0.002 \\
\hline
\end{tabular}

WBC White blood cel

*Comparison was made using Mann-Whitney $U$ test at $P<0.05$, and these values were described by median with inter-quartile range (25th and 75 th percentile)

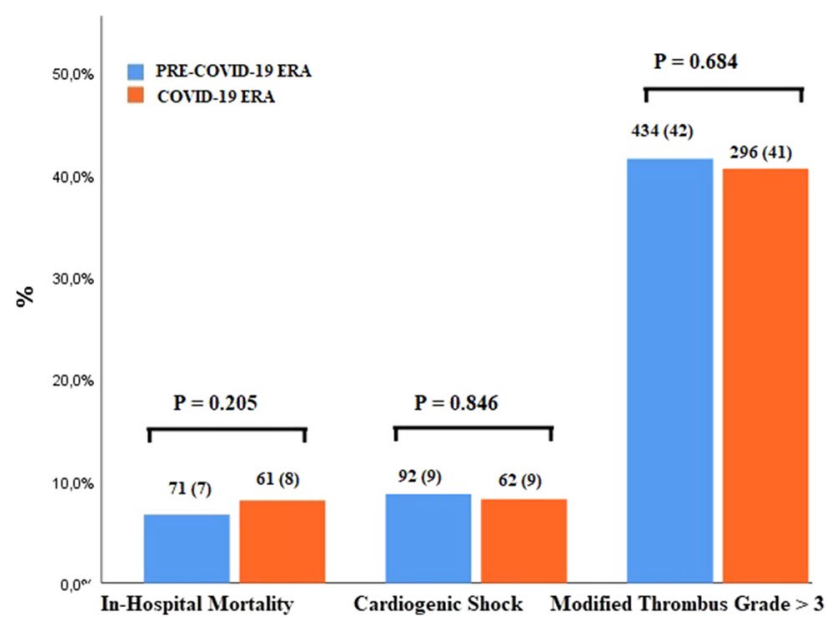

Fig. 4 In-hospital outcomes include mortality, cardiogenic shock and baseline thrombus grade for pre-COVID-19 era and COVID-19 era

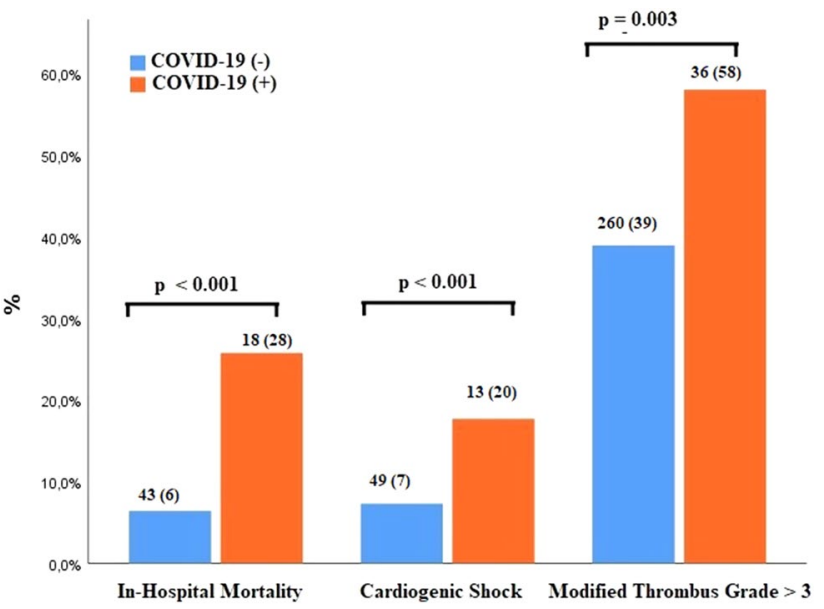

Fig. 5 In-hospital outcomes include mortality, cardiogenic shock and baseline thrombus grade for non-COVID and COVID groups before matching 
Fig. 6 In-hospital outcomes include mortality, cardiogenic shock and baseline thrombus grade for non-COVID and COVID groups after matching

Fig. 7 Positive correlation between thrombus grade and d-dimer levels in COVID-19 positive STEMI patients
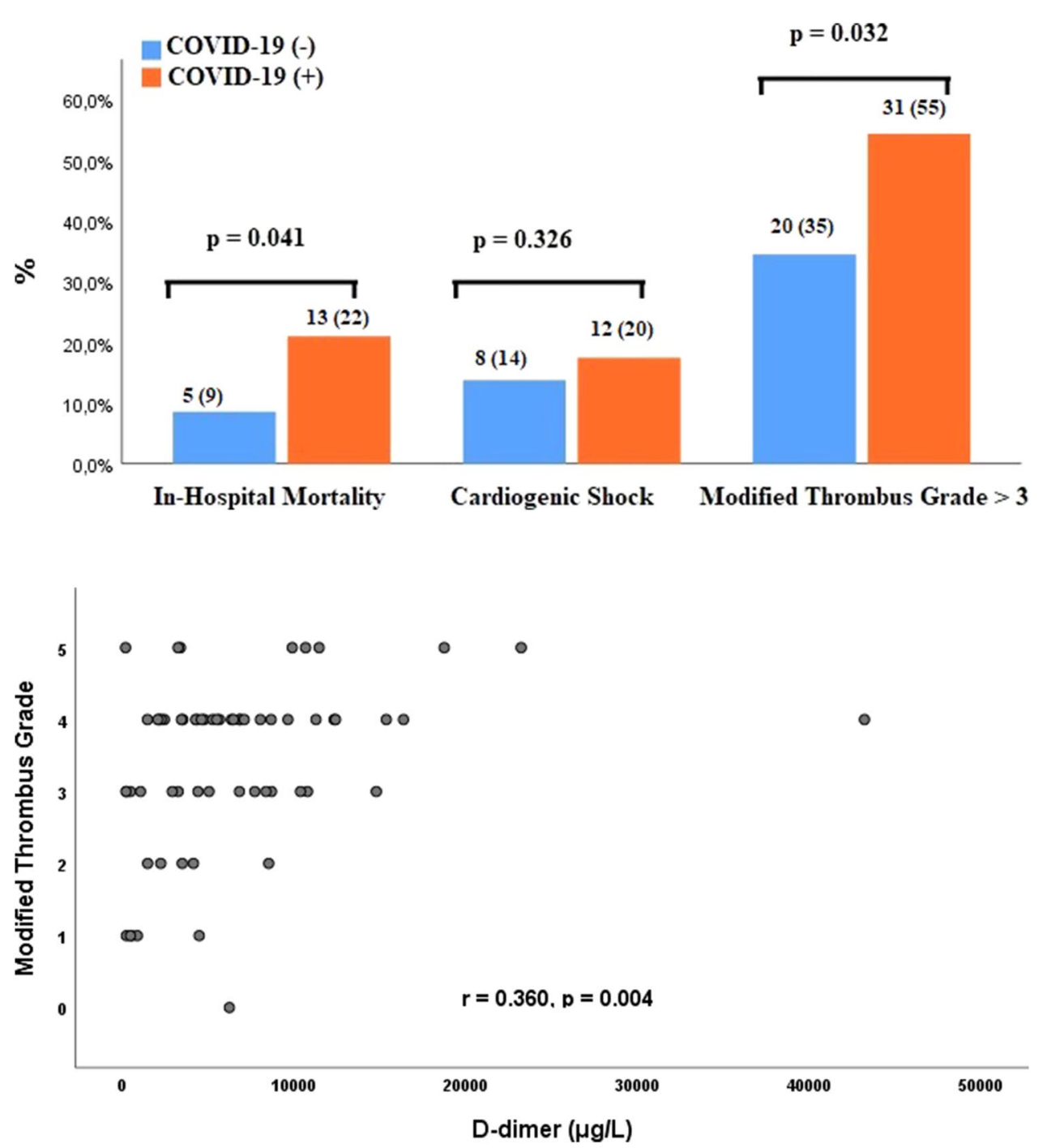

(Fig. 6). Also, in this group, modified thrombus grade was positively correlated with D-dimer levels $(r=0.360$, $\mathrm{p}=0.004$, Fig. 7). Post-PCI TIMI 3 flow was similar in both groups. LVEF was significantly lower in the COVID19 positive patients than COVID-19 negative patients (Tables 3, and 4). Complete revascularization during the index hospitalization was performed in $29 \%$ of COVID-19 patients $(p=0.021)$. Stent thrombosis was more common in COVID-19 patients than COVID-19 negative patients $(6 \%$ vs. $1 \%, \mathrm{p}=0.002$ ).

\section{In-hospital outcomes}

In hospital mortality and shock rates were similar between COVID-19 era and pre-COVID-19 era (7\% vs. $8 \%$, $\mathrm{p}=0.205 ; 9 \%$ vs. $9 \%, \mathrm{p}=0.846$, respectively, Fig. 4).
Patients with STEMI who were infected with COVID19 had higher mortality and cardiogenic shock rates than those without COVID-19 (28\% vs. 6\%, p $<0.001 ; 20 \%$ vs. $7 \%, \mathrm{p}<0.001$, respectively, Fig. 5). The presence of valve disease in COVID-19 positive STEMI patients was linked to much higher mortality compared with those did not $(23 \%$ vs $7 \%, \mathrm{p}=0.035)$.

Matching based on propensity scores (supplementary Fig. 1, Tables 5 and 6) produced 59 patients in each group and showed that mortality rate $(22 \%$ vs. $9 \%, p=0.041)$, stent thrombosis rate $(7 \%$ vs. $0 \%, \mathrm{p}=0.042)$, and modified thrombus grade $(55 \%$ vs. $35 \%, \mathrm{p}=0.032)$ were high in COVID-19 group (Fig. 6). Although cardiogenic shock was more common in COVID-19 positive STEMI patients, this was not statistically significant ( $20 \%$ vs. $14 \%, \mathrm{p}=0.326)$ (Table 5). 
Table 5 Baseline characteristics of the study population after matching

\begin{tabular}{|c|c|c|c|}
\hline Variables & Non-COVID-19 $(\mathrm{n}=59)$ & COVID-19 $(\mathrm{n}=59)$ & p-value \\
\hline Age, years & $68.1 \pm 11.8$ & $66.1 \pm 12.0$ & 0.368 \\
\hline Symptoms at admission n (\%) & & & 0.346 \\
\hline Chest pain & $34(58)$ & $34(58)$ & \\
\hline Dyspnea & $18(31)$ & $13(22)$ & \\
\hline Arrest & $0(0)$ & $0(0)$ & \\
\hline Other & $7(12)$ & $12(20)$ & \\
\hline Female gender $(\%)$ & $21(36)$ & $20(31)$ & 0.847 \\
\hline Hypertension, n (\%) & $30(51)$ & $29(49)$ & 0.854 \\
\hline Diabetes mellitus, n (\%) & $21(36)$ & $16(27)$ & 0.321 \\
\hline Previous AF, n (\%) & $7(12)$ & $3(5)$ & 0.186 \\
\hline Smoking, $\mathrm{n}(\%)$ & $15(25)$ & $20(34)$ & 0.314 \\
\hline COPD or asthma, n (\%) & $12(20)$ & $9(15)$ & 0.470 \\
\hline Previous CAD, n (\%) & $8(14)$ & $11(19)$ & 0.452 \\
\hline \multicolumn{4}{|l|}{ Echocardiographic findings } \\
\hline $\operatorname{LVEF}(\%)$ & $44.6 \pm 10.3$ & $43.8 \pm 8.8$ & 0.644 \\
\hline LVWM abnormalities n (\%) & $42(71)$ & $46(78)$ & 0.398 \\
\hline Valve disease n (\%) & $15(25)$ & $12(20)$ & 0.499 \\
\hline Coronary intervention $\mathrm{n}(\%)$ & $58(98)$ & $57(97)$ & 0.559 \\
\hline Infarct related artery n $(\%)$ & & & 0.978 \\
\hline LMCA & $1(2)$ & $1(2)$ & \\
\hline LAD & $27(47)$ & $22(39)$ & \\
\hline $\mathrm{CX}$ & $12(21)$ & $14(25)$ & \\
\hline $\mathrm{RCA}$ & $12(21)$ & $13(23)$ & \\
\hline Other & $5(9)$ & $6(11)$ & \\
\hline Noncritical CAD & $1(2)$ & $1(2)$ & \\
\hline Multi-vessel disease n (\%) & $26(45)$ & $26(46)$ & 0.932 \\
\hline Glycoprotein IIb/IIIa inhibitors n (\%) & $22(37)$ & $19(32)$ & 0.562 \\
\hline Thrombus aspiration device $\mathrm{n}(\%)$ & $8(14)$ & $9(14)$ & 1.000 \\
\hline IABP n (\%) & $0(0)$ & $2(4)$ & 0.150 \\
\hline \multicolumn{4}{|l|}{ Baseline TIMI flow n (\%) } \\
\hline TIMI flow $0-1$ & $52(90)$ & $53(93)$ & 0.527 \\
\hline Baseline thrombus grade $>3$ & $37(64)$ & $54(95)$ & $<0.001$ \\
\hline Modified thrombus grade $>3$ & $20(35)$ & $31(55)$ & 0.032 \\
\hline Post-PCI TIMI 3 flow n (\%) & $43(74)$ & $48(84)$ & 0.184 \\
\hline Multi-vessel PCI during the index prodecure $\mathrm{n}(\%)$ & $6(11)$ & $4(7)$ & 0.509 \\
\hline $\begin{array}{l}\text { Complete revascularization during the index hospitalization } \\
\mathrm{n}(\%)\end{array}$ & $13(22)$ & $16(27)$ & 0.521 \\
\hline \multicolumn{4}{|l|}{ Previous medication } \\
\hline ACE-I/ARB, n (\%) & $19(32)$ & $17(29)$ & 0.689 \\
\hline Statin n $(\%)$ & $12(20)$ & $14(24)$ & 0.657 \\
\hline Aspirin n (\%) & $27(46)$ & $22(37)$ & 0.350 \\
\hline $\mathrm{P} 2 \mathrm{Y} 12 \mathrm{Y}$ inhibitors & & & 0.573 \\
\hline Clopidogrel n (\%) & $25(42)$ & $22(37)$ & \\
\hline Tigacrelor $\mathrm{n}(\%)$ & $0(0)$ & $0(0)$ & \\
\hline Prasuqrel n (\%) & $0(0)$ & $0(0)$ & \\
\hline In hospital or discharge ASA $+\mathrm{P} 2 \mathrm{Y} 12 \mathrm{Y}$ inhibitors & & & 0.549 \\
\hline ASA plus Clopidogrel n (\%) & $42(73)$ & $34(63)$ & \\
\hline ASA plus Tigacrelor $\mathrm{n}(\%)$ & $11(19)$ & $13(24)$ & \\
\hline ASA plus Prasuqrel n (\%) & $5(9)$ & 7 (13) & \\
\hline Pharmaco-invazive treatment $\mathrm{n}(\%)$ & $0(0)$ & $1(2)$ & 0.315 \\
\hline
\end{tabular}


Table 5 (continued)

\begin{tabular}{|c|c|c|c|}
\hline Variables & Non-COVID-19 $(n=59)$ & COVID-19 $(\mathrm{n}=59)$ & p-value \\
\hline Patients treated with medical treatment $\mathrm{n}(\%)$ & $2(3)$ & $2(5)$ & 0.648 \\
\hline Symptom-to-FMC, minutes (median [IQR]) & $170(90-600)$ & $120(85-240)$ & 0.683 \\
\hline Symptom-to- (FMC) time & & & 0.893 \\
\hline Less than $2 \mathrm{~h}, \mathrm{n}(\%)$ & $20(34)$ & $21(38)$ & \\
\hline 2 to $6 \mathrm{~h}, \mathrm{n}(\%)$ & $10(17)$ & $12(22)$ & \\
\hline 6 to $12 \mathrm{~h}, \mathrm{n}(\%)$ & $23(39)$ & $17(31)$ & \\
\hline 12 to $24 \mathrm{~h}, \mathrm{n}(\%)$ & $2(3)$ & $2(2)$ & \\
\hline More than $24 \mathrm{~h}, \mathrm{n}(\%)$ & $4(7)$ & $3(6)$ & \\
\hline \multicolumn{4}{|l|}{ In-hospital outcomes } \\
\hline Minor bleeding $\mathrm{n}(\%)$ & $4(7)$ & $1(2)$ & 0.170 \\
\hline Major bleeding $\mathrm{n}(\%)$ & $2(3)$ & $0(0)$ & 0.154 \\
\hline Stent thrombosis n (\%) & $0(0)$ & $4(7)$ & 0.042 \\
\hline Cardiogenic shock, n (\%) & $8(14)$ & $12(20)$ & 0.326 \\
\hline Mortality, n (\%) & $5(9)$ & $13(22)$ & 0.041 \\
\hline
\end{tabular}

$A C E-I / A R B$ angiotensin converting enzyme inhibitors/ angiotensin receptor blocker, $A F$ atrial fibrillation, $C A D$ coronary artery disease, $C O P D$ chronic obstructive pulmonary disease, $F M C$ first medical contact, IABP intra aortic balon pump, IQR inter quartile range, $L V E F$ left ventricular ejection fraction, $L V W M$ left ventricular wall motion abnormalities, PCI percutaneous coronary intervention, STEMI ST-segment elevation myocardial infarction

Table 6 Laboratory findings of patients after matching

\begin{tabular}{llll}
\hline Variable & Non-COVID-19 $(\mathrm{n}=59)$ & COVID-19 $(\mathrm{n}=59)$ & p-value \\
\hline WBC $\left(\times 10^{3} / \mu \mathrm{L}\right)$ & $13.2 \pm 6.9$ & $13.5 \pm 4.7$ & 0.787 \\
Hemoglobin $(\mathrm{mg} / \mathrm{dl})$ & $13.3 \pm 2.1$ & $13.7 \pm 2.3$ & 0.267 \\
Creatinine* $(\mathrm{mg} / \mathrm{dl})$ & $0.94(0.78-1.20)$ & $0.90(0.78-1.21)$ & 0.920 \\
Platelet $\left(\times 10^{9} / \mathrm{L}\right)$ & $252.4 \pm 68.3$ & $253.4 \pm 88.4$ & 0.948 \\
C-reactive protein* $(\mathrm{mg} / \mathrm{L})$ & $18.1(2.9-62.0)$ & $54.0(20.0-120)$ & 0.027 \\
Troponin & $14,370(2500-35,056)$ & $18,892(6261-26,842)$ & 1.000 \\
\hline
\end{tabular}

WBC White blood cell

*Comparison was made using Mann-Whitney $U$ test at $P<0.05$, and these values were described by median with inter-quartile range (25th and 75 th percentile)

\section{Discussion}

The presented study showed that there was a significant decrease in hospitalization rates for STEMI during COVID19 pandemic in Turkey. Compared with pre COVID era, we found a 30.5\% drop in STEMI admission with significant delay in symptoms to-FMC time. Also, LVEF was found to be lower in this period than in pre-COVID era in these patients. Furthermore, we found that COVID-19 positive STEMI patients had an increased thrombus burden as demonstrated by high thrombus grade with increased thrombus aspiration device, and glycoprotein IIb/IIIa inhibitors. Although there was no significant difference with regard to symptom onset to- FMC time between groups, these patients had a great myocardial damage with lower LVEF and higher levels of troponin. Also, the rate of mortality was more common in COVID-19 positive STEMI patients.

US cardiac catheterization laboratories reported 38\% reduction in STEMI admission [13]. Similarly, a 40\% reduction was noticed in Spain [14]. STEM admission reduction was reported as $33 \%$ in United Kingdom, and as $26.5 \%$ in Italy $[2,15]$. In presented study, we showed a significant decrease (a 30.5\% drop) in STEMI hospitalization during COVID-19 compared to pre-COVID-19 era as found in TURKMI-2 registry [16]. The mechanisms leading to the reduction in admissions for myocardial infarction remain unknown. Fear of contagion in the hospital or transmitting infection to their close contacts may be the reasons for decreased application to the emergency room. During the COVID-19 pandemic, many countries including Spain, UK, China witnessed significantly reduced rates of house 
containment, factory exhaust, land and air traffic. This led to a marked reduction in air pollution/nitrogen oxide, which is considered as an important risk factor for cardiovascular disease $[17,18]$. The reduction in air pollution may have resulted in a decrease in plaque rupture and MI during this period. Fast food meal consumption was shown to be associated with endothelial dysfunction that acute impairs vasodilatory dysfunction assessed by reduced brachial artery flow-mediated dilatation [19]. The decreased occurrence of STEMI may be associated with reduced access to fast food and processed foods as the resturants were closed in this period. It has been shown that long working hours might increase the risk of cardiovascular disease [20]. Also, healthy lifestyle was found to be associated with reduced premature mortality and prolong life expectancy [21, 22]. Shortening working hours, relaxed lifestyle, improvement in mental and physical health, less work-related stress in COVID-19 pandemic may reduce triggering of plaque rupture via positive effects of these on hemodynamic fluctuations, heart rhythm, and vasomotion abnormality. These ideas are mostly hypothetical. Thus, the aforementioned associations do not reveal exact mechanism of the decline in admission of STEMI patients to the hospital in our country.

Total ischemic time is the most important determinant of cardiovascular outcomes in STEMI patients. It has been shown that mortality rates are increased as this time these patients [23]. Recent studies on the management of STEMI during the COVID-19 pandemic have shown that time from the onset of symptoms to the first medical contact was an average of $318 \mathrm{~min}$ [1]. In another study, this duration was found to be as 227 (65-790) minutes [15]. In presented study, we found that the time in symptom-to-FMC during the COVID-19 era was longer than pre-COVID-19 era in STEMI. As time from FMC to coronary angiography was not evaluated in our study, we did not have a information regarding system related delays in presented study. However, patient-related delay was significantly increased in this study. In presented study, in-hospital mortality rate was similar between COVID-19 and pre-COVID-19 era as found in study of Abdelaziz HK et al. [15]. Also, another study published by Tam et al. showed that there was no difference between patients in COVID-19 era and those in preCOVID-19 era regarding to mortality rates [24]. A recent meta-analysis showed that the covid-19 pandemic was not associated with increased in-hospital mortality [25].

There is limited data on COVID-19 positive STEMI patients [6]. COVID-19 has been shown to be associated with prothrombotic state in previous studies [25-29]. The mechanisms associated with high thrombus burden in STEMI patients who were COVID-19 positive are unknown. A systemic inflammatory response causing endothelial and haemostatic activation, including activation of platelets and the coagulation cascade were observed more commonly in SARS-CoV-2 infection [30]. It has been found that the increased atherosclerotic plaque rupture risk is linked to myocardial infarction because of the profound inflammatory response and haemodynamic changes in acute respiratory infections with influenza virüs [31]. In presented study, COVID-19 positive patients had higher levels of WBC and $\mathrm{CRP}$ as markers of higher inflammatuar response. These findings were similar to results of the previos study published by Choudry et al. In their study, CRP level was higher, and lymphocyte count was lower in STEMI patients who were infected by SARS-COV-2.

High thrombus grade was more common in COVID-19 positive patients as found in the study carried out by Choudry [6]. As there was no significant difference in terms of symptom onset-to FMC time and culprit vessel between groups, high thrombus grade may be the result of increased activation of platelets, coagulation system and inflammation leading to endotelial dysfunction in COVID-19 positive STEMI patients. Also, the presence of a positive association between thrombus grade and d-dimer levels may be a reflection enhanced COVID-19 related thrombogenicity [6].

In presented study, although in-hospital mortality and shock rates did not differ between pandemic era and prepandemic era as in the recently published meta-analysis [25], these patients may have a new diagnosis of heart failure which is associated with higher mortality in long-term follow-up. However, COVID-19 positive STEMI patiens had higher rates of in-hospital mortality when compared to those without COVID-19. Although symptom onset to-FMC, culprit vessel, risk factors such as DM, HT except age, valve disease, COPD or asthma were similar between groups, coronary intervention rate was lower in those with COVID-19 with STEMI. All these factors, high thrombotic burden, the presence of cardiogenic shock, higher myocardial damage with high troponin level and lower LVEF, and COVID-19 itself leading to endotelial dysfunction and increased immun response may be causes of poor outcomes in these patients.

\section{Limitations}

The presented study has several limitations. This study has a retrospective nature. As the patients in this study were only followed up in hospital during COVID-19 pandemic, we have limited data on the impact of this outbreak on long-term mortality in STEMI patients. Since the onset of symptoms is a subjective parameter, this might not be precisely recorded. The number of patients who were received pharmaco-invasive treatment was very low in presented study. Therefore, we did not compared impact of the pharmaco-invasive treatment with primary PCI in-hospital outcomes in these patients. Also, the sensitivity of diagnostic 
test for SARS-CoV-2 is modest at approximately $60-70 \%$ with nasal/pharyngeal swab as is universally accepted [7]. 53 patients included in this study were enrolled in the other study published by Erol et al. [16].

\section{Conclusions}

In COVID-19 era, there was a significant reduction in STEMI patients admission in our country. Also, COVID-19 outbreak may lead to delays in treatment of these patients. Furthermore, COVID-19 positive STEMI patients represent high risk patients population as thrombus grade and mortality rate were higher in these patients. Thus, during the current COVID-19 outbreak, doctors and health care providers should inform the public and increase awareness with regard to myocardial infarction symptoms, encourage them to apply to the hospital and receive treatment on time. Also, it is important to know COVID-19 status in all STEMI patients. Primary PCI may be preferable and feasible in these patients with semptom onset to FMC time similar to COVID-19 negative. Moreover, these patients population may need more agressive anti-thrombotic treatment. Further studies are needed to reveal exact mechanism of coronary thrombus load, determine treatment, and impact of COVID19 on poor outcomes in these patients.

Supplementary Information The online version contains supplementary material available at https://doi.org/10.1007/s11239-021-02487-3.

Author contributions All authors contributed to the final manuscript.

Funding This research received no grant from any funding agency in the public, commercial, or not-for-provit sectors.

Data availability The data underlying this article will be shared on reasonable request to the corresponding author.

\section{Declarations}

Conflict of interest The author(s) declared no potential conflicts of interest with respect to the research, authorship, and/or publication of this article.

\section{References}

1. Onder G, Rezza G, Brusaferro S (2020) Case-fatality rate and characteristics of patients dying in relation to COVID-19 in Italy. JAMA 323(18):1775-1776

2. De Rosa $\mathrm{S}$, Spaccarotella $\mathrm{C}$, Basso $\mathrm{C}$, SocietàItaliana di Cardiologia and the CCU Academy investigators group et al (2020) Reduction of hospitalizations for myocardial infarction in Italy in the COVID-19 era. Eur Heart J 41(22):2083-2088

3. Nallamothu BK, Normand S-LT, Wang Y et al (2015) Relation between door-to balloon times and mortality after primary percutaneous coronary intervention over time: a retrospective study. Lancet 385(9973):1114-1122

4. Guerchicoff A, Brener SJ, Maehara A et al (2014) Impact of delay to reperfusion on reperfusion success, infarct size, and clinical outcomes in patients with ST segment elevation myocardia linfarction: the INFUSE-AMI trial (INFUSE anterior myocardial infarction). JACC Cardiovasc Interv 7(7):733-740

5. Bikdeli B, Madhavan MV, Jimenez D et al (2020) COVID-19 and thrombotic or thromboembolic disease: implications for prevention, antithrombotic therapy, and follow-up. J Am Coll Cardiol 75:2950-2973

6. Choudry FA, Hamshere SM, Rathod KS et al (2020) High thrombus burden in patients with COVID-19 presenting with ST-elevation myocardial infarction. J Am Coll Cardiol. https://doi.org/10.1016/j. jacc.2020.07.022

7. Hartikainen TS, So “rensen NA, Haller PM et al (2020) Clinical application of the 4th universal definition of myocardial infarction. Eur Heart J 41(23):2209-2216

8. Ibanez B, James S, Agewall S et al (2018) 2017 ESC Guidelines for the management of acute myocardial infarction in patients presenting with ST-segment elevation: the task force for the management of acute myocardial in. Eur Heart J 00(2):119-177

9. Sianos G, Papafaklis MI, Serruys PW (2010) Angiographic thrombus burden classification in patients with ST-segment elevation myocardial infarction treated with percutaneous coronary intervention. $\mathrm{J}$ Invasive Cardiol 22:6B-14B

10. Gibson CM, de Lemos JA, Murphy SA, Marble SJ, McCabe CH, Cannon CP, Antman EM, Braunwald E (2001) Combination therapy with abciximab reduces angiographically evident thrombus in acute myocardial infarction: a TIMI 14 substudy. Circulation 103:2550-2554

11. Patel A, Jernigan DB (2020) Initial public health response and interim clinical guidance for the 2019 novel coronavirus outbreakUnited States, December 31, 2019-February 4, 2020. MMWR Morb Mortal Wkly Rep 69(5):140-146

12. Simpson S, Kay FU, Abbara S et al (2020) Radiological Society of North America expert consensus statement on reporting chest CT findings related to COVID-19. Endorsed by the Society of Thoracic Radiology, the American College of Radiology, and RSNA. J Thorac Imaging. https://doi.org/10.1177/0846537120938328

13. Garcia S, Albaghdadi MS, Meraj PM et al (2020) Reduction in ST segment elevation cardiac catheterization laboratory activations in the United States during COVID-19 pandemic. J Am Coll Cardiol 75(22):2871-2872

14. Rodriguez-Leor O, Cid-Alvarez B, Ojeda S et al (2020) Impacto de la pandemia de COVID-19 sobre la actividad asistencialen cardiologia intervencionista en Espana. REC Interv Cardiol 155(4):179-180

15. Abdelaziz HK, Abdelrahman A, Nabi A et al (2020) Impact of COVID-19 pandemic on patients with ST-segment elevation myocardial infarction: insights from a British cardiac center. Am Heart J 226:45-48

16. Erol MK, Kayıkçıŏlu M, Kılıçkap M et al (2020) Treatment delays and in-hospital outcomes in acute myocardial infarction during the COVID-19 pandemic: a nationwide study. Anatol J Cardiol 24(5):334-342

17. Brook RD, Sanjay Rajagopalan C, Pope A et al (2010) Particulate matter air pollution and cardiovascular disease. Circulation 121(21):2331-2378

18. PhilipJ Landrigan R, Fuller NA et al (2018) The lancet commission on pollution and health. Lancet 391(10119):462-512

19. Patik JC, Tucker WJ, Curtis BM, Nelson MD, Nasirian A, Park S, Brothers RM (2018) Fast-food meal reduces peripheral artery endothelial function but not cerebralvascular hypercapnic reactivity in healthy young men. Physiol Rep 6(18):e13867

20. Kivimäki M, Jokela M, Nyberg ST, IPD-Work Consortium et al (2015) Long working hours and risk of coronary heart 
disease and stroke: a systematic review and meta-analysis of published and unpublished data for 603,838 individuals. Lancet 386(10005): 1739-46

21. Nyberg ST, Singh-Manoux A, Pentti J et al (2020) Association of healthy lifestyle with years lived without major chronic diseases. JAMA Intern Med 180(5):1-10

22. Li Y, Pan A, Wang DD et al (2018) Impact of healthy lifestyle factors on life expectancies in the US population. Circulation 138(4):345-355

23. De Luca G, Suryapranata H, Ottervanger JP, Antman EM (2004) Time delay to treatment and mortality in primary angioplasty for acute myocardial infarction: every minute of delay counts. Circulation 109(10):1223-1225

24. Tam CF, Cheung KS, Lam S et al (2020) Impact of corona virüs disease 2019 COVID-19 outbreak on ST-segment elevation myocardial infarction care in Hong-Kong, China. Circ Cardiovasc Qual Outcomes. https://doi.org/10.1002/ccd.28943

25. Levi M, Thachil J, Iba T, Levy JH (2020) Coagulation abnormalities and thrombosis in patients with COVID-19. Lancet Haematol 7:e438-e440

26. Fan BE, Chong VCL, Chan SSW et al (2020) Hematologic parameters in patients with COVID-19 infection. Am J Hematol 95:e131-e134
27. Klok FA, Kruip MJHA, van der Meer NJM et al (2020) Incidence of thrombotic complications in critically ill ICU patients with COVID19. Thromb Res 191:145-147

28. Tang N, Li D, Wang X, Sun Z (2020) Abnormal coagulation parameters are associated with poorprognosis in patients with novel coronavirus pneumonia. J Thromb Haemost 18:844-847

29. Beyrouti R, Adams ME, Benjamin L et al (2020) Characteristics of ischaemic stroke associated with COVID-19. J Neurol Neurosurg Psychiatry 91(8):889-891

30. Masi P, Hekimian G, Lejeune M et al (2020) Systemic inflammatory response syndrome is a major contributor to COVID-19-associated coagulopathy: insights from a prospective single center cohort study. Circulation 142(6):611-614. https://doi.org/10.1161/circulationaha. 120.048925

31. Kwong JC, Schwartz KL, Campitelli MA et al (2018) Acute myocardial infarction after laboratory-confirmed influenza infection. $\mathrm{N}$ Engl J Med 378:345-353

Publisher's Note Springer Nature remains neutral with regard to jurisdictional claims in published maps and institutional affiliations.

\section{Authors and Affiliations}

Tuncay Kiris ${ }^{1}$ - Eyüp Avci ${ }^{2}$ Tuba Ekin ${ }^{3}$. Didar Elif Akgün ${ }^{2}$ - Mücahit Tiryaki ${ }^{1}$ - Arafat Yidirim ${ }^{4} \cdot$ Kutluhan Hazir $^{5}$. Bektaş Murat $^{6} \cdot$ Mehtap Yeni $^{7} \cdot$ Rojhad Altindag $^{8} \cdot$ Sefa Gül ${ }^{9} \cdot$ Baran Arik $^{10}$. Tuncay Güzel $^{11}$. Selda Murat ${ }^{12}$. Ahmet $\mathrm{Oz}^{13} \cdot$ Mustafa Karabacak $^{14} \cdot$ Zihni Aktas $^{15} \cdot$ Tarik Yildirim $^{2} \cdot$ Baris Kilicaslan $^{5} \cdot$ Asim Oktay Ergene $^{3}$

1 Department of Cardiology, Ataturk Training and Research Hospital, Izmir Katip Celebi University, Izmir, Turkey

2 Department of Cardiology, Balikesir University Medical School, Balikesir, Turkey

3 Department of Cardiology, Dokuz Eylul University Faculty of Medicine, İzmir, Turkey

4 Department of Cardiology, Ministry Of Health Adana City Training \& Research Hospital, Adana, Turkey

5 Department of Cardiology, Izmir University Of Health Sciences Tepecik Training And Research Hospital, Izmir, Turkey

6 Department of Cardiology, Ministry Of Health Eskisehir City Hospital, Eskisehir, Turkey

7 Department of Cardiology, Ministry Of Health Isparta City Hospital, Isparta, Turkey

8 Department of Cardiology, Training and Research Hospital, Health Sciences University Diyarbakır Gazi Yasargil, Diyarbakır, Turkey
9 Department of Cardiology, Samsun Education and Research Hospital, Samsun, Turkey

10 icle University Medical School, Dicle University, Diyarbakır, Turkey

11 Department of Cardiology, Akhisar State Hospital, Manisa, Turkey

12 Department of Cardiology, Faculty of Medicine, Eskisehir Osmangazi University, Eskisehir, Turkey

13 Department of Cardiology, Istanbul Education and Research Hospital, Istanbul, Turkey

14 Department of Cardiology, Faculty of Medicine, Suleyman Demirel University, Isparta, Turkey

15 Department of Cardiology, BalikesirAtatürk City Hospital, Balikesir, Turkey 